\title{
Snakes With an Ellipse-Reproducing Property
}

\author{
Ricard Delgado-Gonzalo, Philippe Thévenaz, Chandra Sekhar Seelamantula, Member, IEEE, and \\ Michael Unser, Fellow, IEEE
}

\begin{abstract}
We present a new class of continuously defined parametric snakes using a special kind of exponential splines as basis functions. We have enforced our bases to have the shortest possible support subject to some design constraints to maximize efficiency. While the resulting snakes are versatile enough to provide a good approximation of any closed curve in the plane, their most important feature is the fact that they admit ellipses within their span. Thus, they can perfectly generate circular and elliptical shapes. These features are appropriate to delineate cross sections of cylindrical-like conduits and to outline bloblike objects. We address the implementation details and illustrate the capabilities of our snake with synthetic and real data.
\end{abstract}

Index Terms-Active contour, exponential B-spline, parameterization, parametric snake, segmentation.

\section{INTRODUCTION}

A CTIVE contours and, in particular, snakes are effective tools for image segmentation. Within an image, an active contour is a curve that evolves from an initial position, which is usually specified by a user, toward the boundary of an object. The evolution of the curve is formulated as a minimization problem. The associated cost function is called snake energy. Snakes have become popular because it is possible for the user to interact with them not only when specifying its initial position but also during the segmentation process.

Research in this area has been fruitful and has resulted in many snake variants [1], [2]. They differ in the type of curve representation and in the choice of the energy term [3]. Snakes can be broadly categorized in terms of curve representation as follows:

1) point-snakes, where the curve is described in a discrete fashion by a set of points [4]-[6];

2) parametric snakes, where the curve is described continuously by some coefficients using basis functions [7]-[11];

3) implicit snakes, where the representation of the curve is implicit and described as the level set of a surface [12]-[15].

Point-snakes can be viewed as a special case of parametric snakes where a large number of coefficients is used [10].

Manuscript received March 23, 2011; revised July 20, 2011 and September 12, 2011; accepted September 19, 2011. Date of publication September 29, 2011; date of current version February 17, 2012. This work was supported in part by Swiss SystemsX.ch under Grant 2008/005 and in part by the Swiss National Science Foundation under Grant 200020-121763. The associate editor coordinating the review of this manuscript and approving it for publication was Prof. Ferran Marques.

R. Delgado-Gonzalo, P. Thévenaz, and M. Unser are with the Biomedical Imaging Group, École polytechnique fédérale de Lausanne, 1015 Lausanne, Switzerland.

C. S. Seelamantula is with the Department of Electrical Engineering, Indian Institute of Science, Bangalore 560 012, India.

Color versions of one or more of the figures in this paper are available online at http://ieeexplore.ieee.org.

Digital Object Identifier 10.1109/TIP.2011.2169975

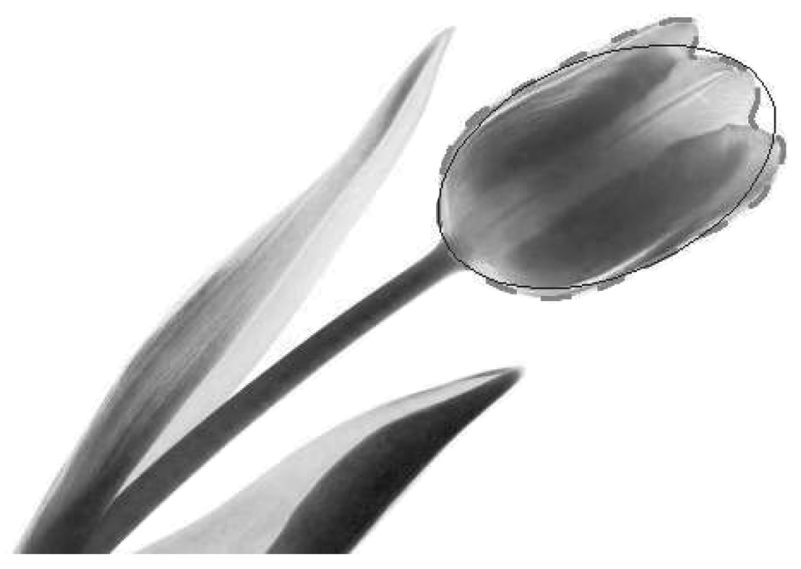

Fig. 1. Approximation capabilities of the proposed parametric snake. The thin solid line corresponds to an elliptical fit. The dashed thick line corresponds to a generalized shape.

Parametric snakes require fewer parameters and result in faster optimization. It can be shown that the computation complexity of the snake energy and, therefore, the speed of the optimization algorithms is related to the size of the support of the basis functions [3]. It is therefore critical to minimize this support while designing parametric snakes. The curve of parametric snakes is represented explicitly. It is then easy to introduce smoothness and shape constraints [7]. It is also straightforward to accommodate user interaction. This is often achieved by allowing the user to specify some anchor points that the curve should go through [4]. The downsize of the method is that the topology of the curve is imposed by the parameterization. This makes parametric snakes less suitable for handling topological changes, although solutions have been proposed for specific cases [16], [17].

Implicit approaches offer great flexibility as far as the curve topology is considered [18]. However, they tend to be computationally more expensive since they evolve a $2-\mathrm{D}$ surface rather than a 1-D curve.

In this paper, we design fast parametric snakes capable of perfectly outlining elliptic objects and yet versatile enough to provide a close approximation of any closed curve in the plane. We illustrate in Fig. 1 how our snake can adopt the shape of a perfect ellipse (i.e., reproduces the ellipse) as well as more refined shapes. Segmenting circles and ellipses in images is a problem that arises in many fields, such as biomedical engineering [19]-[22] or computer graphics [23], [24]. In medical imaging in particular, it is usually necessary to segment arteries and veins within tomographic slices [25]. Because those objects are physiological tubes, their section show up as ellipses in the image. Ellipse-like objects are also present at microscopic scales. For instance, cell nuclei are known to be nearly circular 
[26]. Water drops are similarly spherical due to surface tension forces [27]. However, these elements deform and become elliptical when they are subject to stress forces.

In order to segment efficiently elliptical objects, a parametric snake called the Ovuscule was proposed in [28]. It is a minimalistic elliptical snake defined by three control points. Its main drawback was that it was unable to represent shapes different from circles and ellipses. Our goal here is to create a more versatile parametric snake whose basis functions are short, reproduce ellipses perfectly, and have good approximation properties. Our main contribution in this paper is to fulfill this goal by selecting a special kind of exponential B-splines. We are able to prove that our basis functions are the ones with the shortest support among all admissible functions. Since the computational cost of spline snakes is determined in part by the size of the support of the basis function, our use of the shortest possible support favors optimal performance.

This paper is organized as follows. In Section II, we review the general parametric snake model and formalize our design constraints. Our main contribution is described in Section III, where we build an explicit expression for the underlying basis functions that fulfill our requirements, and we analyze in detail its reproduction and approximation properties. Implementation details such as energy functionals and discretization issues are addressed in Section IV. Finally, we perform report evaluations in Section V.

\section{PARAMETRIC SNAKES}

\section{A. Parametric Representation of Closed Curves}

Curve $\mathbf{r}(t)$ on the plane can be described by a pair of Cartesian coordinate functions $x_{1}(t)$ and $x_{2}(t)$, where $t \in \mathbb{R}$ is a continuous parameter. The 1-D functions $x_{1}$ and $x_{2}$ are efficiently parameterized by linear combinations of suitable basis functions. Among all possible bases, we focus on those derived from the compactly supported generator $\varphi$ and its integer shifts

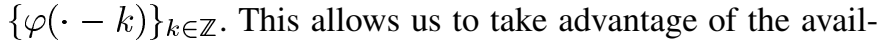
ability of fast and stable interpolation algorithms [29].

We are interested in close curves specified by an $M$-periodic sequence of control points $\{\mathbf{c}[k]\}_{k \in \mathbb{Z}}$, with $\mathbf{c}[k]=\mathbf{c}[k+M]$. The parametric representation of the curve is then given by the following vectorial equation:

$$
\mathbf{r}(t)=\sum_{k=-\infty}^{\infty} \mathbf{c}[k] \varphi(M t-k) .
$$

The number of control points $M$ determines the degrees of freedom in model (1). Small numbers lead to constrained shapes and large numbers lead to additional flexibility and more general shapes.

Since curve $\mathbf{r}$ is closed, each coordinate function is periodic, and the period is common for both. For simplicity, in (1), we normalized this period to be unity. Under these conditions, we can reduce the infinite summation in (1) to a finite one involving periodized basis functions as

$$
\mathbf{r}(t)=\sum_{k=0}^{M-1} \sum_{n=-\infty}^{\infty} \mathbf{c}[M n+k] \varphi(M(t-n)-k)
$$

$$
=\sum_{k=0}^{M-1} \mathrm{c}[k] \underbrace{\sum_{n=-\infty}^{\infty} \varphi(M(t-n)-k)}_{\varphi_{M}(M t-k)}
$$

where $\varphi_{M}$ is the $M$-periodization of the basis function $\varphi$.

This kind of curve parameterization is general. Using this model, we can approximate any closed curve as accurately as desired by using a higher number of vector coefficients $M_{2}>$ $M$, under the condition $\varphi$ satisfies some mild conditions [30].

\section{B. Desirable Properties for the Basis Functions}

We now enumerate the conditions that our parametric snake model should satisfy and introduce the corresponding mathematical formalism.

1) Unique and Stable Representation. We want our parametric curve to be defined in terms of the coefficients in such a way that unicity of representation is satisfied. Furthermore, for computational purposes, we ask the interpolation procedure to be numerically stable.

The generating function $\varphi$ is said to satisfy the Riesz basis condition if and only if there exist two constants $0<A \leq$ $B<\infty$ such that

$A\|\mathbf{c}\|_{\ell_{2}} \leq \sqrt{M}\left\|\sum_{k=-\infty}^{\infty} \mathbf{c}[k] \varphi(M \cdot-k)\right\|_{L_{2}} \leq B\|\mathbf{c}\|_{\ell_{2}}$

for all $\mathbf{c} \in \ell_{2}$. A direct consequence of the lower inequality is that condition $\sum_{k=-\infty}^{\infty} \mathbf{c}[k] \varphi(M t-k)=0$ for all $t \in \mathbb{R}$ implies that $\mathbf{c}[k]=0$ for all $k \in \mathbb{Z}$. Thus, the basis functions are linearly independent, and every function is uniquely specified by its coefficients. The upper inequality ensures the stability of the interpolation process [29].

It has been shown in [31] that, due to the integer-shift-invariant structure of the representation, the Riesz condition has the following equivalent expression in the Fourier domain:

$$
A \leq \sum_{k=-\infty}^{\infty}|\hat{\varphi}(\cdot+2 \pi k)|^{2} \leq B
$$

where $\hat{\varphi}(\omega)=\int_{\mathbb{R}} \varphi(x) \mathrm{e}^{-\mathrm{j} \omega x} d x$ denotes the Fourier transform of $\varphi$. Once expressed in the Fourier domain, the Riesz condition provides a practical way to verify if a given generating function $\varphi$ satisfies (3).

2) Affine Invariance. Since we are interested in outlining shapes irrespective of their position and orientation, we would like our model to be invariant to affine transformations, which we formalize as

$$
\mathbf{A r}(t)+\mathbf{b}=\sum_{k=-\infty}^{\infty}(\mathbf{A c}[k]+\mathbf{b}) \varphi(M t-k)
$$

where $\mathbf{A}$ is a $(2 \times 2)$ matrix and $\mathbf{b}$ is a 2 -D vector. Using (4), it is easy to show that affine invariance is ensured if and only if

$$
\forall t \in \mathbb{R}: \sum_{k=-\infty}^{\infty} \varphi(M t-k)=1 .
$$




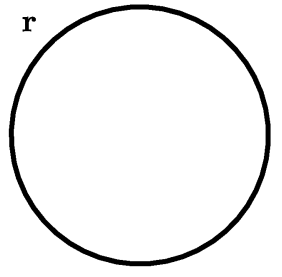

(a)

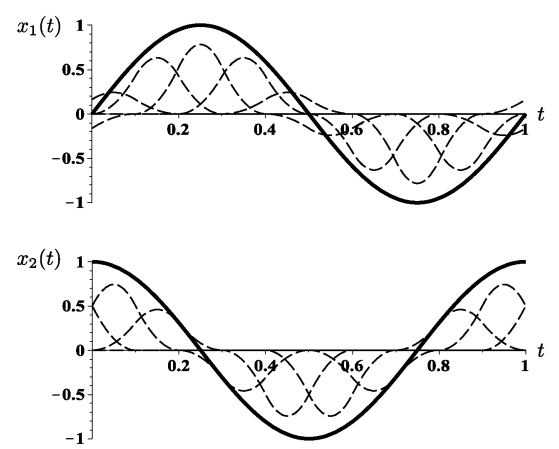

(b)
Fig. 2. (a) Parametric representation of the unit circle and (b) its coordinate functions with exponential B-splines and $M=10$. The dashed lines in (b) indicate the corresponding basis functions.

In the literature, this constraint is often called the partition-of-unity condition [29].

3) Well-Defined Curvature. The curvature of a parametric curve at point $\left(x_{1}(t), x_{2}(t)\right)$ is given by

$$
\kappa\left(x_{1}, x_{2}\right)=\frac{\dot{x}_{1} \ddot{x}_{2}-\ddot{x}_{1} \dot{x}_{2}}{\left(\dot{x}_{1}^{2}+\dot{x}_{2}^{2}\right)^{3 / 2}}
$$

where the dot denotes the derivative with respect to $t$. We would like to be able to compute $\kappa$ for every point on the snake. To do so, each coordinate function (or, equivalently, basis $\varphi$ ) must be at least twice differentiable, and its second derivative must be bounded.

\section{REPRODUCTION OF ELLIPSES}

Since every ellipse can be obtained by applying an affine transformation to the unit circle, we focus on the reproduction of this simpler shape. This simplification is allowed whenever the affine-invariance requirement stated in Section II-B is satisfied.

A parametric snake defined by $M$ vectorial coefficients and by a generating function $\varphi$ is said to reproduce the unit circle if there exist two $M$-periodic sequences $\left\{c_{1}[k]\right\}_{k \in \mathbb{Z}}$ and $\left\{c_{2}[k]\right\}_{k \in \mathbb{Z}}$ such that

$$
\begin{aligned}
& \cos (2 \pi t)=\sum_{k=-\infty}^{\infty} c_{1}[k] \varphi(M t-k) \\
& \sin (2 \pi t)=\sum_{k=-\infty}^{\infty} c_{2}[k] \varphi(M t-k) .
\end{aligned}
$$

That is, we need to be able to reproduce sinusoids of unit period for each component of the parametric snake, as illustrated in Fig. 2. Note that, when (6) and (7) hold, it is possible to represent any sinusoid of unit period for an arbitrary initial phase using linear combinations of the two sequences of coefficients.

\section{A. Minimum-Support Ellipse-Reproducing Basis}

We now present and prove our main result. We provide an explicit expression for the minimum-support basis functions that reproduce sinusoids.
Theorem 1: The centered generating function with minimal support that satisfies all conditions in Section II-B and reproduces sinusoids of unit period with $M$ coefficients is

$$
\varphi(t)= \begin{cases}\frac{\cos \frac{2 \pi|t|}{M} \cos \frac{\pi}{M}-\cos \frac{2 \pi}{M}}{1-\cos \frac{2 \pi}{M}}, & 0 \leq|t|<\frac{1}{2} \\ \frac{1-\cos \frac{2 \pi(3 / 2-|t|)}{M}}{2\left(1-\cos \frac{2 \pi}{M}\right)}, & \frac{1}{2} \leq|t|<\frac{3}{2} \\ 0, & \frac{3}{2} \leq|t| .\end{cases}
$$

In order to prove Theorem 1, we refer to the distributional decomposition theorem detailed in [32]. This decomposition theorem provides a complete characterization of the family of basis functions with minimum support that reproduce exponential polynomials. It states that every minimum-support function $\varphi$ that reproduces exponentials $e^{\alpha_{n} t}$, for all $n \in[0 \ldots N-1]$ with $\alpha_{i}-\alpha_{j} \notin 2 \pi \mathrm{j} \mathbb{Z}$, can be written as

$$
\varphi(t)=\sum_{n=0}^{N-1} \lambda_{n} \frac{d^{n}}{d t^{n}} \beta_{\boldsymbol{\alpha}}(t-a)
$$

where $a$ is an arbitrary shift parameter that corresponds to the lower extremity of the support of $\varphi$ and where $\beta_{\boldsymbol{\alpha}}$ is the appropriate exponential B-spline defined as follows:

$$
\hat{\beta}_{\boldsymbol{\alpha}}(\omega)=\prod_{n=1}^{N} \frac{1-\mathrm{e}^{\alpha_{n}-\mathrm{j} \omega}}{\mathrm{j} \omega-\alpha_{n}} .
$$

Note that exponential B-splines are entirely specified by collection $\boldsymbol{\alpha}=\left(\alpha_{1}, \ldots, \alpha_{N}\right)$. The ordering of the poles $\alpha_{n}$ is irrelevant. A complete survey of the properties of exponential B-splines can be found in [33].

We finally have the mathematical tools to justify our choice for the generating function in (8).

Proof: Using (9), we see that $\varphi$ needs to be constructed from combinations of exponential B-splines with parameters $\boldsymbol{\alpha}=(0, \mathrm{j}(2 \pi / M),-\mathrm{j}(2 \pi / M))$ and $N=3$. Therefore, we have

$$
\varphi(t)=\sum_{n=0}^{2} \lambda_{n} \frac{d^{n}}{d t^{n}} \beta_{\boldsymbol{\alpha}}(t-a) .
$$

This ensures that $\varphi$ is the shortest generating function that reproduces constants and all sinusoids of unit period with $M$ coefficients. The constant-reproduction property is a direct consequence of using $\alpha_{0}=0$, and the sinusoid-reproduction property can be proved by using $\alpha_{1}=\mathrm{j}(2 \pi / M), \alpha_{2}=-\mathrm{j}(2 \pi / M)$, and Euler's identity.

Using the properties of exponential B-splines, we know that $\beta_{\boldsymbol{\alpha}}$ is twice differentiable. Moreover, the second derivative is bounded but may be discontinuous. Therefore, $\lambda_{1}$ and $\lambda_{2}$ in (11) must vanish to ensure that the curvature of the snake is well defined. Since $\varphi$ reproduces constants, $\lambda_{0}$ can be computed by imposing the partition-of-unity condition. From (5), we have

$$
\lambda_{0}=\frac{\left(\frac{2 \pi}{M}\right)^{2}}{2\left(1-\cos \frac{2 \pi}{M}\right)} .
$$

Exponential B-splines parameterized by $\boldsymbol{\alpha}$ form a Riesz basis if and only if $\alpha_{m_{1}}-\alpha_{m_{2}} \notin 2 \pi \mathrm{j} \mathbb{Z}$ for all pairs such that $m_{1} \neq$ $m_{2}$. In our case, it is important to realize that this condition is satisfied if and only if $M \geq 3$. In other words, at least three control points are needed to define our parametric snake. 


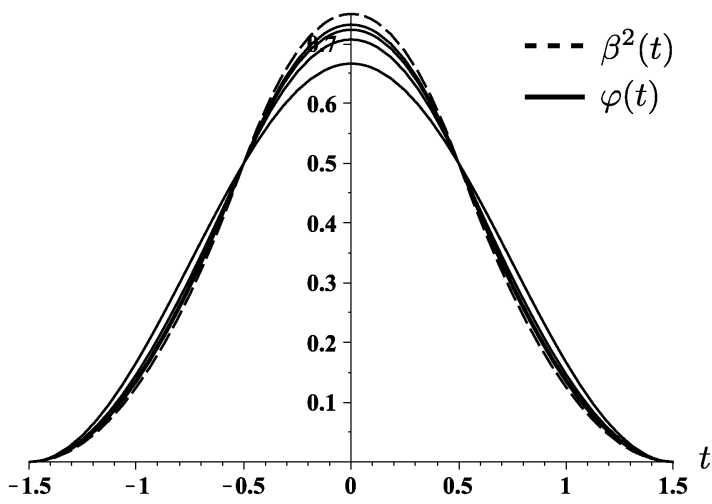

Fig. 3. Plot of the quadratic B-spline $\beta^{2}$ and the resulting generating functions given in (8) for $M=3,4,5$, and 6 . The function with the lowest peak at $t=0$ corresponds to $M=3$, and as $M$ increases, the height of the central peak increases as well.

Finally, a closed form for $\varphi$ is obtained by applying the inverse Fourier transform to (11), which yields

$$
\hat{\varphi}(\omega)=\lambda_{0} \mathrm{e}^{\mathrm{j} \frac{3 \omega}{2}} \frac{1-\mathrm{e}^{-\mathrm{j} \omega}}{\mathrm{j} \omega} \frac{1-\mathrm{e}^{\mathrm{j} \frac{2 \pi}{M}-\mathrm{j} \omega}}{\mathrm{j} \omega-\mathrm{j} \frac{2 \pi}{M}} \frac{1-\mathrm{e}^{-\mathrm{j} \frac{2 \pi}{M}-j \omega}}{\mathrm{j} \omega+\mathrm{j} \frac{2 \pi}{M}}
$$

where we have set $a=-(3 / 2)$ in order to ensure that the basis function is centered.

We show in Fig. 3 some members of this family of functions for several values of $M$. We observe that they share with the quadratic B-spline a finite support of length $W=3$. Likewise, they are one-time continuously differentiable and have a similar bumplike appearance.

\section{B. Approximation Properties of $\varphi$}

We are not only interested in reproducing ellipses but would also like our snake to be able to approximate any other shape $\mathbf{s}$. This is achieved by increasing the number of degrees of freedom afforded by the number $M$ of nodes. In the Fourier domain, it is easy to see that $\varphi$ converges to a quadratic $\mathrm{B}$-spline as $M$ increases. Therefore, we expect similar approximation properties for large values of $M$.

While $\varphi$ leads to integer-shift invariance, the space spanned by the generating function $\varphi$ is not shift invariant in general. Hence, the approximation error using $M$ vector coefficients is dependent upon a shift in the continuous parameter $t$ of the function of unit period $\mathbf{s}$. The minimum-mean-square approximation error for a shifted function is given by

$$
\begin{aligned}
\gamma(\tau, M) & =\int_{0}^{1}\|\mathbf{s}(t-\tau)-\mathbf{r}(t)\|^{2} d t \\
& =\|\mathbf{s}(\cdot-\tau)-\mathbf{r}(\cdot)\|_{L_{2}([0,1])}^{2}
\end{aligned}
$$

where $\mathbf{r}$ is the best approximation within the span $\{\varphi(M$. $-k)\}_{k \in \mathbb{Z}}$. Since $\tau$ is usually unknown, we measure the error averaged over all possible shifts as

$$
\eta(M)=\left(\int_{0}^{1} \gamma(\tau, M) d \tau\right)^{\frac{1}{2}} .
$$

We give in Section III-C the decay of $\eta$ as $M \rightarrow \infty$, following the method described in [30]. As expected, we find that the best averaged-quadratic-mean error decays as $1 / M^{3}$ when the number of vector coefficients $M$ increases, which results in the same rate as the quadratic B-spline [34].

\section{Approximation Order of $\varphi$}

In this section, we introduce the necessary formalism to compute the order of the approximation error associated to the best possible approximation of the periodic vector function $\mathbf{s}$ within the span of basis $\{\varphi(M \cdot-k)\}_{k \in \mathbb{Z}}$, where $\varphi$ is given by (8).

As explained in Section III-B about the approximation properties of $\varphi$, the space spanned by the generating function $\varphi$ is not shift invariant in general. Hence, as a metric of dissimilarity between shapes, we use the averaged minimum-mean-square approximation error $\eta$.

Using the main result of [30], we obtain the asymptotic behavior of $\eta$ as

$$
\begin{aligned}
\eta^{2}(M)= & C_{1}^{2}(M)\|\dot{\mathbf{s}}\|_{L_{2}([0,1])}^{2} M^{-2} \\
& +C_{2}^{2}(M)\|\dot{\mathbf{s}}\|_{L_{2}([0,1])}^{2} M^{-4}+\mathcal{O}\left(M^{-6}\right)
\end{aligned}
$$

where $C_{L}=(1 / L !) \sqrt{\left(\sum_{k \neq 0}\left|\hat{\varphi}^{(L)}(2 \pi k)\right|^{2}\right)}$, and $\hat{\varphi}^{(L)}$ is the $L$ th derivative of the Fourier transform of $\varphi$. Following lengthy calculations, we get (13) and (14), shown at the bottom of the page, where we defined $M_{0}=\pi \cot (\pi / M)$. It can be shown that $C_{1}(M)=\mathcal{O}\left(M^{-2}\right)$ and $C_{2}(M)=\mathcal{O}\left(M^{-2}\right)$. Since curve $\mathbf{s}$ does not depend on $M$, we can also write that

$$
\eta(M)=\left(\mathcal{O}\left(M^{-6}\right)\right)^{\frac{1}{2}}=\mathcal{O}\left(M^{-3}\right)
$$

which shows that the averaged quadratic mean error decays as $M^{-3}$.

\section{Best Constant and Ellipse Fitting}

Since our snakes have the capability of perfectly reproducing ellipses, it is natural to ask which is the best ellipse that approximates the parametric curve $\mathbf{r}$ defined by the $M$-periodic se-

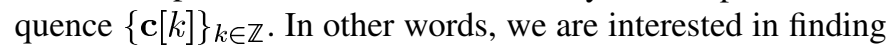
ellipse $\mathbf{r}_{e}$ that minimizes

$$
\left\|\mathbf{r}-\mathbf{r}_{e}\right\|_{L_{2}([0,1])}^{2}=\int_{0}^{1}\left\|\mathbf{r}(t)-\mathbf{r}_{e}(t)\right\|^{2} d t .
$$

$$
\begin{aligned}
& C_{1}(M)=\frac{1}{12 \pi} \sqrt{18\left(M_{0}-M\right)\left(M_{0}+4 M\right)+30 \pi^{2}} \\
& C_{2}(M)=\frac{1}{120 \pi^{2}} \sqrt{225\left(2 M_{0}^{4}-7 M^{2} M_{0}^{2}-15 M^{3} M_{0}+20 M^{4}\right)+75\left(8 M_{0}^{2}-29 M^{2}\right) \pi^{2}+170 \pi^{4}}
\end{aligned}
$$


Since $\mathbf{r}$ is continuous and has a unit period, we can expand it in a Fourier series as

$$
\mathbf{r}(t)=\sum_{n=-\infty}^{\infty} \mathbf{R}[n] \mathrm{e}^{\mathrm{j} 2 \pi n t}
$$

The Fourier series vector coefficients $\mathbf{R}$ in (15) are given by

$$
\begin{aligned}
\mathbf{R}[n] & =\int_{0}^{1} \mathbf{r}(t) \mathrm{e}^{-\mathrm{j} 2 \pi n t} d t \\
& =\frac{1}{M} \hat{\varphi}\left(\frac{2 \pi n}{M}\right) \sum_{k=0}^{M-1} \mathbf{c}[k] \mathrm{e}^{-\mathrm{j} \frac{2 \pi}{M} n k}
\end{aligned}
$$

where the parametric expression of $\mathbf{r}$ has been used in the second equality.

From the classical theory of harmonic analysis, we know that the best ellipse approximation (componentwise sinusoids) of $\mathbf{r}$, in the $L_{2}([0,1])$ sense, is the first-order truncation of the series (15), where only the terms $n=-1, n=0$, and $n=1$ are kept. Therefore, we have

$$
\begin{aligned}
\mathbf{r}_{e}(t)= & \mathbf{R}[0]+(\mathbf{R}[1]+\mathbf{R}[-1]) \cos (2 \pi t) \\
& +\mathrm{j}(\mathbf{R}[1]-\mathbf{R}[-1]) \sin (2 \pi t)
\end{aligned}
$$

where $\mathbf{R}[0]$ is the center of gravity of the snake. The Fourier coefficients in (17) can be obtained easily from (16) as

$$
\begin{aligned}
\mathbf{R}[0] & =\frac{1}{M} \sum_{k=0}^{M-1} \mathbf{c}[k] \\
\mathbf{R}[1]+\mathbf{R}[-1] & =\sum_{k=0}^{M-1} h_{c}[k] \mathbf{c}[k] \\
\mathbf{j}(\mathbf{R}[1]-\mathbf{R}[-1]) & =\sum_{k=0}^{M-1} h_{s}[k] \mathbf{c}[k]
\end{aligned}
$$

where

$$
\begin{aligned}
& h_{c}[k]=\frac{2}{M} \cos \frac{\pi}{M} \cos \frac{2 \pi k}{M} \\
& h_{s}[k]=\frac{2}{M} \cos \frac{\pi}{M} \sin \frac{2 \pi k}{M} .
\end{aligned}
$$

Since all sinusoids of unit period can be reproduced by the generating function $\varphi$ and the appropriate $M$-periodic sequence of coefficients $\mathbf{c}$, curve $\mathbf{r}_{e}$ belongs to the span of $\varphi$. For the sake of completeness, we provide in the next section an explicit expansion of sinusoids in terms of $\varphi$.

\section{E. Expansion of Sinusoids With $\varphi$}

Here, we explicitly find the sequence of $M$ vector coefficients that reproduce sinusoids of unit period using the generating function $\varphi$ given in (8). We start by recalling the exponential-reproducing property of the exponential B-splines as

$$
\mathrm{e}^{\alpha t}=\sum_{k=-\infty}^{\infty} \mathrm{e}^{\alpha k} \beta_{(\alpha)}(t-k) .
$$

Setting $\alpha=\mathrm{j}(2 \pi / M)$, we see that $\beta_{(\mathrm{j}(2 \pi / M))}$ reproduces the complex exponential $\mathrm{e}^{\mathrm{j}(2 \pi / M) t}$, which is $M$-periodic. If we now convolve both sides of (18) with $\beta_{(0,-\mathrm{j}(2 \pi / M))}$, we get that

$$
\begin{aligned}
\left(\beta_{\left(0,-\mathrm{j} \frac{2 \pi}{M}\right)} * \mathrm{e}^{\mathrm{j} \frac{2 \pi}{M}}\right) & (t) \\
& =\sum_{k=-\infty}^{\infty} \mathrm{e}^{\mathrm{j} \frac{2 \pi}{M} k} \underbrace{\left(\beta_{\left(\mathrm{j} \frac{2 \pi}{M}\right) * \beta\left(0,-\mathrm{j} \frac{2 \pi}{M}\right)}\right)(t-k)}_{\frac{2\left(1-\cos \frac{2 \pi}{M}\right)}{\left(\frac{2 \pi}{M}\right)^{2}} \varphi\left(t-\frac{3}{2}-k\right)}
\end{aligned}
$$

where we have used the definition of $\varphi$ from (8), along with the fact that the convolution operator commutes with the shift operator. To simplify the left-hand side, we invoke an important property of linear shift-invariant (LSI) systems: complex exponentials are eigenfunctions of LSI operators. By virtue of this property, if the complex exponential $\mathrm{e}^{\mathrm{j} \alpha t}$ is presented at the input of a system specified by the impulse response $h$, then its output is given by $\hat{h}(\alpha) \mathrm{e}^{\mathrm{j} \alpha t}$, where $\hat{h}$ denotes the Fourier transform of $h$. If we consider $\beta_{(0,-j(2 \pi / M))}$ as the impulse response of an LSI system, then

$$
\left(\beta_{\left(0,-\mathrm{j} \frac{2 \pi}{M}\right)} * \mathrm{e}^{\mathrm{j} \frac{2 \pi}{M} \cdot}\right)(t)=\left.\underbrace{\hat{\beta}}_{\lambda}\left(0,-\mathrm{j} \frac{2 \pi}{M}\right)(\omega)\right|_{\omega=\frac{2 \pi}{M}} \mathrm{e}^{\mathrm{j} \frac{2 \pi}{M} t} .
$$

Therefore, we have that

$$
\mathrm{e}^{\mathrm{j} \frac{2 \pi}{M} t}=\sum_{k=-\infty}^{\infty} \mathrm{e}^{\mathrm{j} \frac{2 \pi}{M} k} 2 \frac{1-\cos \frac{2 \pi}{M}}{\lambda\left(\frac{2 \pi}{M}\right)^{2}} \varphi\left(t-\frac{3}{2}-k\right)
$$

By flipping the sign of $\alpha$ we can easily obtain an analogous result for the reproduction of $\mathrm{e}^{-\mathrm{j}(2 \pi / M) t}$. Finally, by using both results, we have

$$
\begin{aligned}
& \cos \left(2 \pi\left(t+\frac{3}{2 M}\right)\right)=\sum_{k=-\infty}^{\infty} c_{1}[k] \varphi(M t-k) \\
& \sin \left(2 \pi\left(t+\frac{3}{2 M}\right)\right)=\sum_{k=-\infty}^{\infty} c_{2}[k] \varphi(M t-k)
\end{aligned}
$$

where

$$
\begin{aligned}
& c_{1}[k]=\frac{2\left(1-\cos \frac{2 \pi}{M}\right) \cos \frac{\pi(2 k+3)}{M}}{\cos \frac{\pi}{M}-\cos \frac{3 \pi}{M}} \\
& c_{2}[k]=\frac{2\left(1-\cos \frac{2 \pi}{M}\right) \sin \frac{\pi(2 k+3)}{M}}{\cos \frac{\pi}{M}-\cos \frac{3 \pi}{M}} .
\end{aligned}
$$

Note that sequences $c_{1}$ and $c_{2}$ are $M$-periodic and that the summations in (19) and (20) can be reduced to finite ones if we make use of the periodized basis functions.

We have expressed in (19) and (20) how to compute the vector coefficients for reproducing sinusoids of unit period and the initial phase of $3 \pi / M$. The appropriate linear combination of $c_{1}$ and $c_{2}$ then allows one to reproduce sinusoids of arbitrary shape. 


\section{IMPLEMENTATION}

Since the presented parametric active contour is a spline snake, it is capable of handling all traditional energies applicable to point and parametric snakes. However, to illustrate the behavior of our parameterization in a real implementation, we performed our experiments with a specific snake energy that we designed to be versatile.

In this section, we first introduce the snake energy that drives the optimization process, and then, we provide a description of the implementation details for the proposed snake. We construct the energy functional to detect dark objects on a brighter background.

\section{A. Snake Energy}

The active-contour algorithm is always driven by a chosen energy function. Thus, the quality of the segmentation depends on the choice of this energy term. There are many construction strategies that can be categorized in two main families: 1) edge-based schemes, which use gradient information to detect contours [4], [7], [10]; and 2) region-based methods, which use statistical information to distinguish different homogeneous regions [9], [35]. In order to benefit from the advantages of both strategies, a unified energy was proposed in [3]. In our case, we are going to follow a similar approach by using a convex combination of gradient energy and region energy, such as in

$$
E=\alpha E_{\text {edge }}+(1-\alpha) E_{\text {region }}
$$

where $\alpha \in[0,1]$. The tradeoff parameter $\alpha$ balances the contribution of the edge-based energy and the region-based energy. Its value depends on the characteristics of each particular application.

For the gradient-based (or edge) energy, we consider the one described in [35] since it has the advantage of penalizing the snake when the orientation is inconsistent with the object to segment. Let $\mathbf{r}$ be our parametric snake. The contour energy term is then given by

$$
E_{\text {edge }}=-\oint_{\mathbf{r}} \mathbf{k}^{T}\left(\nabla f\left(x_{1}, x_{2}\right) \times d \mathbf{x}\right)
$$

where $d \mathbf{x}$ denotes the tangent vector of the curve in the 3-D space formed by the image plane and its orthogonal dimension, $\mathbf{k}=(0,0,1)$ denotes the outward vector orthonormal to the image plane, $\nabla f\left(x_{1}, x_{2}\right)=$ $\left(\left(\partial f\left(x_{1}, x_{2}\right) / \partial x_{1}\right),\left(\partial f\left(x_{1}, x_{2}\right) / \partial x_{2}\right), 0\right)$ is the within-plane gradient of image $f$ at $\left(x_{1}, x_{2}\right)$ on the curve, and $\times$ is the 3-D cross product. In Fig. 4, we present the configuration of the various quantities involved. The chirality of the system of coordinates will determine the sign of the integrand, as discussed in [3] and [35]. Using Green's theorem, the edge energy can be also expressed as the surface integral

$$
E_{\text {edge }}=-\int_{\Omega} \Delta f(\mathbf{x}) d x_{1} d x_{2}
$$

where $\mathbf{x}=\left(x_{1}, x_{2}\right), \Delta f$ is the Laplacian of the image $f$, and $\Omega$ is the region enclosed by $\mathbf{r}$.

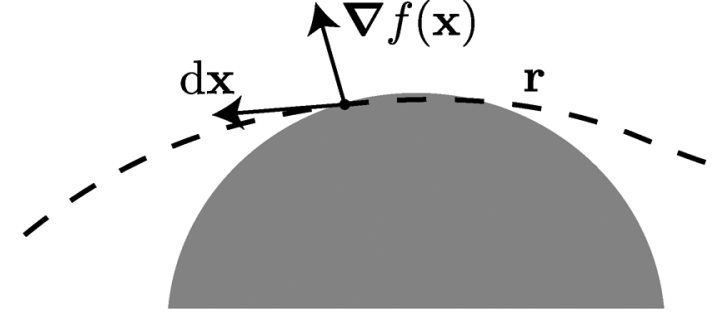

Fig. 4. Schematic representation of a parametric snake $\mathbf{r}$ (dashed line), of its interaction with an object constituted by a gray semicircle (representing low pixel values), of the vector $d \mathbf{x}$ tangent to the curve, and of the gradient vector $\nabla f$ of the image. The vector $\mathbf{k}$, which is mentioned in the text, is perpendicular to the image plane and points toward the reader.

For the region-based energy, we adopt a strategy similar to [28]. More precisely, our region-based energy discriminates an object from its background by building ellipse $\mathbf{r}_{\lambda}$ around the snake and by maximizing the contrast between the intensity of the data averaged within the curve and the intensity of the data averaged over the elliptical shell $\Omega_{\lambda}$. When $\Omega \subset \Omega_{\lambda}$, the region energy term can be expressed as

$$
E_{\text {region }}=\frac{1}{|\Omega|}\left(\int_{\Omega} f(\mathbf{x}) d x_{1} d x_{2}-\int_{\Omega_{\lambda} \backslash \Omega} f(\mathbf{x}) d x_{1} d x_{2}\right)
$$

where $|\Omega|$ is given by

$$
|\Omega|=-\sum_{k=0}^{M-1} \sum_{n=0}^{M-1} c_{1}[k] c_{2}[n] \int_{0}^{M} \varphi_{M}(t-n) \dot{\varphi}_{M}(t-k) d t .
$$

The normalization factor $|\Omega|$ can be interpreted as the signed area, which is defined as $|\Omega|=-\oint_{\mathbf{r}} x_{2} d x_{1}$. The sign of quantity $|\Omega|$ depends on the clockwise or the anticlockwise path followed on curve $\mathbf{r}$. In this paper, we follow the usual convention whereby an anticlockwise path leads to a positive sign. We enforce our criterion to remain neutral $\left(E_{\text {region }}=0\right)$ when $f$ takes a constant value, such as in flat regions of the image. To achieve this, we set $\left|\Omega_{\lambda}\right|=2|\Omega|$.

The construction of the elliptic shell is performed by using the best ellipse $\mathbf{r}_{e}$ given in (17) and by magnifying its axes by factor $\lambda$ to achieve

$$
\begin{aligned}
\mathbf{r}_{\lambda}(t)= & \mathbf{R}[0]+\lambda(\mathbf{R}[1]+\mathbf{R}[-1]) \cos (2 \pi t) \\
& +\mathbf{j} \lambda(\mathbf{R}[1]-\mathbf{R}[-1]) \sin (2 \pi t)
\end{aligned}
$$

where $\lambda=\sqrt{2|\Omega| /\left|\Omega_{e}\right|}$, and $\left|\Omega_{e}\right|$ is the signed area enclosed by curve $\mathbf{r}_{e}$, with

$$
\left|\Omega_{e}\right|=-\frac{4 \pi}{M^{2}} \cos \frac{\pi}{M} \sum_{k=0}^{M-1} \sum_{n=0}^{M-1} c_{1}[k] c_{2}[n] \sin \frac{2 \pi(n-k)}{M} .
$$

In Fig. 5, we illustrate how we take advantage of the ideas presented in Section III-D to build the best ellipse approximation $\mathbf{r}_{e}$ of an arbitrary snake $\mathbf{r}$. Using constraint $\left|\Omega_{\lambda}\right|=2|\Omega|$, we can determine the contour $\mathbf{r}_{\lambda}$ of the enclosing shell $\Omega_{\lambda}$. 


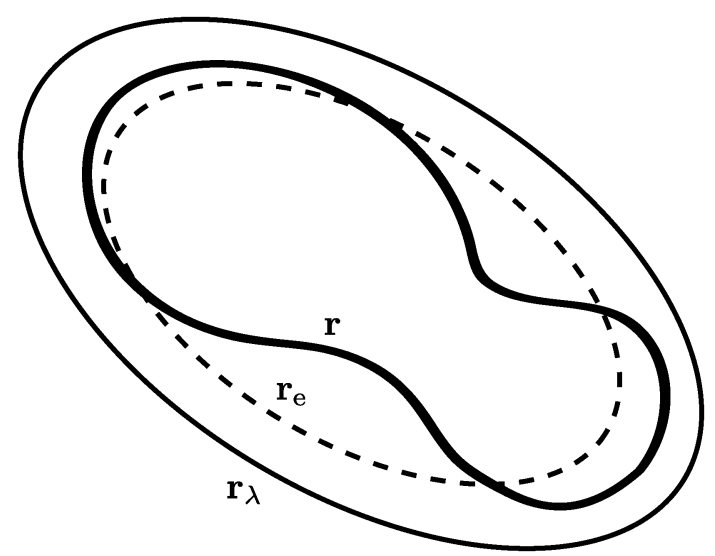

Fig. 5. Representation of the parametric snake $\mathbf{r}$, the best ellipse approximation $\mathbf{r}_{e}$, and the corresponding enclosing shell $\mathbf{r}_{\lambda}$ used in $E_{\text {region }}$.

\section{B. Accelerated Implementation}

The computational cost is dominated by the evaluation of the surface integrals in (22) and (23). An efficient way to implement these operations is the use of preintegrated images. Let $g$ be the function that we are integrating $(\Delta f, f$, or $-f$, respectively) and let $\Gamma$ be the domain of integration $\left(\Omega\right.$ or $\Omega_{\lambda}$ ). Then, by Green's theorem, we rewrite the surface integrals as the line integrals

$$
\begin{aligned}
\int_{\Gamma} g(\mathbf{x}) d x_{1} d x_{2} & =-\oint_{\partial \Gamma} g_{2}\left(x_{1}, x_{2}\right) d x_{1} \\
& =\oint_{\partial \Gamma} g_{1}\left(x_{1}, x_{2}\right) d x_{2}
\end{aligned}
$$

where $\partial \Gamma$ is the boundary of $\Gamma$ and

$$
\begin{aligned}
& g_{2}\left(x_{1}, x_{2}\right)=\int_{-\infty}^{x_{2}} g\left(x_{1}, \xi_{2}\right) d \xi_{2} \\
& g_{1}\left(x_{1}, x_{2}\right)=\int_{-\infty}^{x_{1}} g\left(\xi_{1}, x_{2}\right) d \xi_{1} .
\end{aligned}
$$

The use of Green's theorem to rewrite the surface integrals as line integrals reduces dramatically the computational load. This can only be achieved if the curve is defined continuously, such as with the curves of Section II-A. By contrast, this acceleration would not be available to methods such as point snakes and level sets because their implementation ultimately relies on discretization.

\section{Sampling}

Despite the fact that we are assuming a continuously defined model for our functions, in a real-world implementation we only have at our disposal a sampled version of the functions we want to preintegrate. To solve this inconsistency, we perform a bilinear interpolation of the sampled data, and we store in lookup tables the values of (24) and (25) at integer locations. Then, the energies are obtained using the first approximation given by the lookup tables. In our implementation, we have corrected them by supplying a residual that allows us to get the exact result. We

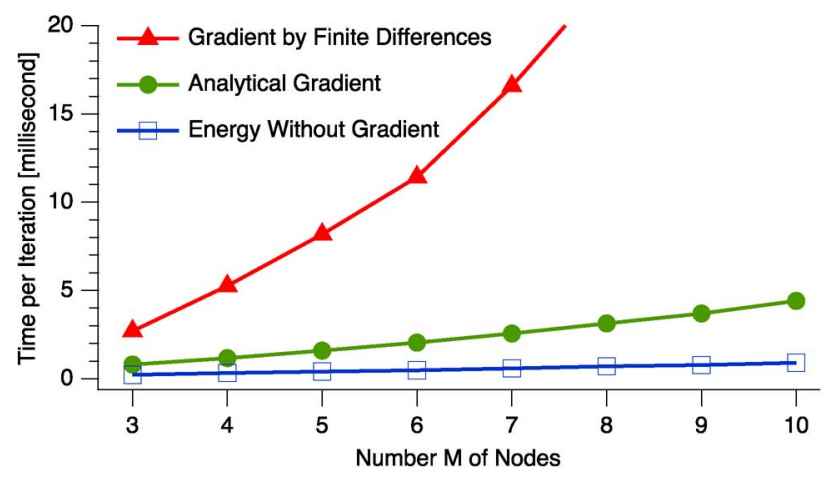

Fig. 6. Mean time of one iteration in the snake evolution.

were able to determine this residual analytically, but in the interest of space, we do not provide it here.

\section{Optimization}

The optimization of the snake can be efficiently carried out by Powell-like line-search methods [36]. These methods require the derivatives of the energy function with respect to the parameters (i.e., the knot coefficients) and converge quadratically to the solution. The algorithm proceeds as follows. First, one direction within the parameter space is chosen depending on the partial derivatives of the energy. Second, 1-D minimization is performed within the selected direction. Finally, a new direction is chosen using the partial derivatives of the energy function once more while enforcing conjugation properties. This scheme is repeated until convergence. Assuming a bilinear interpolation of the original function $f$, we were able to derive exact and closed expressions for these derivatives that take the residual of the lookup table into account.

For spline snakes, it has been shown that the evaluation of the partial derivatives of the energy of form (21) depends quadratically on the number of parameters [3]. In Fig. 6, we compare the computational cost of the snake during line minimization (simple update) and when the energy gradient is required to chose a new direction (gradient update). For the latter case, we contrast the computation time of an analytical computation of the gradient to that of a centered finite-difference approach. For low values of $M$, the simple update and the gradient update using analytical energy gradient lead to a similar computational load. As the value of $M$ increases, the quadratic behavior of the computation of the gradient makes the update cost increase. This quadratic behavior can be easily discerned in the topmost curve of Fig. 6.

\section{EXPERIMENTS}

We present in this section four experimental setups. In the first one, we compare our choice in (8) against the classical quadratic B-spline when representing sinusoids. We move away from sinusoids in the second experiment, where we work with synthetic data and perform an objective validation of the segmentation properties of our snake in noiseless and noisy environments. In the fourth setup, we also perform a quantitative evaluation by segmenting real cardiac MRI data. Finally, in the last experiment, we illustrate some real applications of our snake where the ground truth is not available. 


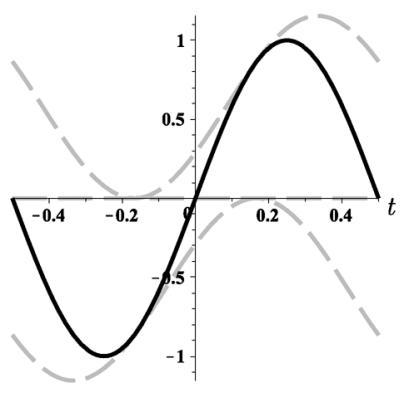

(a)

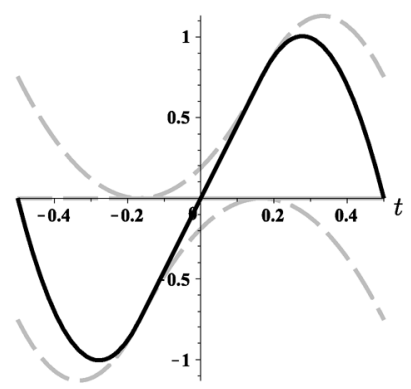

(b)
Fig. 7. Approximations of a sine function with unit period. (a) Parametric representation (solid line) using $\varphi_{3}$ (dashed lines). (b) Best parametric approximation (solid line) using $\beta^{2}$ (dashed lines).

\section{A. Approximation of Sinusoids}

By design, our basis function $\varphi$ has the property of reproducing sinusoids exactly. By contrast, the classical polynomial B-splines do not enjoy this property. In this section, we are focusing on this aspect and exhibit the amount of error committed by B-splines when attempting to reproduce a sine function.

We start with the exact reproduction by our basis. Using the result of Section III-E, we determine the coefficients for case $M=3$ (smallest possible $M$ ). They are given by

$$
\sin (2 \pi t)=\sqrt{3}\left(\varphi_{3}(3 t-1)-\varphi_{3}(3 t+1)\right)
$$

where $\varphi_{3}$ corresponds to the periodization of the basis function (8), as in (2).

We continue with approximate reproduction by B-splines. For fairness, we choose the quadratic B-spline $\beta^{2}$ so that the size of the support of $\beta^{2}$ and $\varphi$ is the same. The reproduction will be approximate. This will happen not because of the limited size of the support but because of the fact that the sine function does not lie in the span of polynomial B-splines of any degree. Nevertheless, we can compute the coefficients that best adjust the sinusoid with unit period in the least-squares sense. This yields

$$
\sin 2 \pi t \approx \frac{1215}{26 \pi^{2}}\left(\beta_{3}^{2}(3 t-1)-\beta_{3}^{2}(3 t+1)\right)
$$

where

$$
\beta^{2}(t)= \begin{cases}\frac{3}{4}-|t|^{2}, & 0 \leq|t|<\frac{1}{2} \\ \frac{1}{2}\left(\frac{3}{2}-|t|\right)^{2}, & \frac{1}{2} \leq|t|<\frac{3}{2} \\ 0, & \frac{3}{2} \leq|t|\end{cases}
$$

is the quadratic B-spline and the subscript 3 indicates a threeperiodized basis function as in (2).

We observe in Fig. 7 that both constructions result in sinelike functions. However, the reproduction is exact in the left part of Fig. 7, whereas it is only approximate in the right part. This happens, despite the fact that the support of $\beta^{2}$ is identical to the support of $\varphi$, the asymptotic approximation properties of $\beta^{2}$ and $\varphi$ are identical, and $\beta^{2}$ and $\varphi$ have the same degree of differentiability. We show in Fig. 8 the amount of error committed by the parabolic approximation. We determine that $\operatorname{MSE}=(1 / 2)-\left(98415 / 208 \pi^{6}\right)$.

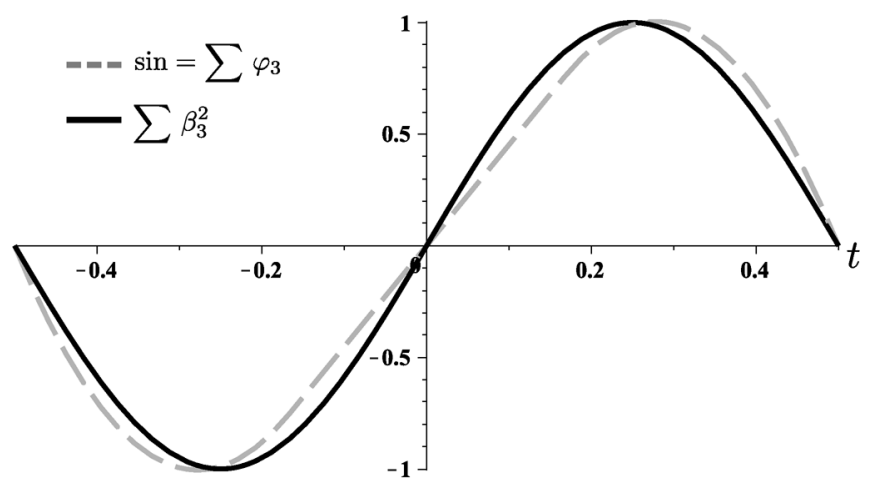

Fig. 8. Sinusoid of period 3, its representation with our basis function (solid line), and its best quadratic B-spline approximation (dashed line).

\section{B. Accuracy and Robustness to Noise}

In this section, two experiments are carried out. The first one consists of outlining different synthetic bloblike shapes in a noise-free environment. The second experiment consists of outlining one specific target within an image in the presence of noise. In both experiments, we set $\alpha=0$, i.e., we make use of the region energy only. This particular choice ensures that the snake is not mislead by noisy boundaries in the presence of excessive of noise.

In the first experiment, we generate ten test images of size 512 $\times 512$ by pixelwise sampling of our shape of interest, which is built by intersecting or by making the union of two circles with a radius of 50 pixel units. We illustrate these shapes in the header of Table I. They are parameterized with distance $d$, in pixel units, between the centers of the circles. For $d<0$, the shape is built by the intersection of the two circles. For $d \geq 0$, they are parameterized by their union. The grayscale values of the images are 255 for the shape and 0 for the background.

We used the Jaccard distance $J=1-|\Theta \cap \Omega| /|\Theta \cup \Omega|$ to measure as a percentage the dissimilarity between the two sets. There, $\Theta$ corresponds to the ground-truth region, and $\Omega$ corresponds to the region enclosed by the snake. We computed $J$ with a pixelwise discretization of the images.

In the simulations of Table I, we investigated the dependence of $J$ on the number $M$ of coefficients and distance $d$ between the circles. We denoted with a dash $(-)$ when the snake did not converge; and, we could not compute the Jaccard distance. We initialized every snake as a circle with a radius of 75 pixel and a center that lay in the middle of the shape. We observe that the results in Table I tend to improve as the number $M$ of control points is increased, particularly for the nonelliptical shapes. However, the increase in the number of control points does not bring any further improvement when the shape to segment is a perfect circle. This result is expected since the circular shape is reproduced exactly for any $M \geq 3$. The residual error shown in Table I for $d=0$ can be attributed to the discretization of $\Theta$ and $\Omega$. We also observe that, for $d=-80$ and $d=-64$, the Jaccard distance starts increasing severely for $M \geq 7$ and for $M \geq 9$, respectively. This is due to the fact that the sharp corners of the shape lead to loops in the curve during the optimization process. Such self-intersections violate the conditions of Green's theorem in Section IV-A. 
TABLE I

ERror Percentage of OUR SNaKe for Noiseless Synthetic Data

\begin{tabular}{c|ccccccccccc}
\hline \hline$M$ & $\mathbf{0}$ & $\mathbf{O}$ & $\mathrm{O}$ & 0 & 0 & 0 & 0 & 0 \\
& $d=-80$ & $d=-64$ & $d=-48$ & $d=-32$ & $d=-16$ & $d=0$ & $d=16$ & $d=32$ & $d=48$ & $d=64$ \\
\hline 3 & 5.08 & 4.12 & 3.78 & 2.84 & 1.54 & 0.17 & 2.18 & 4.06 & 6.63 & 9.49 \\
4 & 4.85 & 4.12 & 3.84 & 2.78 & 1.53 & 0.15 & 2.22 & 4.01 & 6.64 & 9.48 \\
5 & 3.53 & 2.64 & 2.03 & 1.25 & 0.58 & 0.20 & 1.06 & 2.27 & 4.21 & 6.82 \\
6 & 2.69 & 2.18 & 2.00 & 1.13 & 0.64 & 0.17 & 0.91 & 1.81 & 2.84 & 4.36 \\
7 & 3.63 & 1.87 & 1.63 & 1.08 & 0.48 & 0.17 & 1.09 & 1.92 & 2.50 & 3.68 \\
8 & 18.84 & 0.58 & 0.68 & 0.55 & 0.32 & 0.18 & 0.86 & 1.92 & 4.00 & 5.73 \\
9 & - & 1.56 & 0.99 & 0.72 & 0.30 & 0.15 & 0.55 & 0.85 & 1.41 & - \\
10 & - & 1.41 & 0.93 & 0.70 & 0.34 & 0.17 & 0.18 & 0.41 & 0.80 & 1.23 \\
\hline \hline
\end{tabular}

TABLE II

Percentage of Success Rate of OUR SNAKe for Noisy Synthetic Data

\begin{tabular}{|c|c|c|c|c|c|c|}
\hline & $\mathrm{SNR}=15 \mathrm{~dB}$ & $\mathrm{SNR}=10 \mathrm{~dB}$ & $\mathrm{SNR}=5 \mathrm{~dB}$ & $\mathrm{SNR}=0 \mathrm{~dB}$ & $\mathrm{SNR}=-5 \mathrm{~dB}$ & $\mathrm{SNR}=-10 \mathrm{~dB}$ \\
\hline 3 & 100 & 100 & 100 & 100 & 99 & 45 \\
\hline 4 & 100 & 100 & 100 & 100 & 96 & 33 \\
\hline 5 & 99 & 99 & 100 & 100 & 97 & 25 \\
\hline 6 & 100 & 99 & 100 & 99 & 98 & 25 \\
\hline 7 & 100 & 99 & 100 & 99 & 90 & 20 \\
\hline 8 & 99 & 100 & 100 & 98 & 90 & 7 \\
\hline 9 & 99 & 98 & 99 & 96 & 92 & 7 \\
\hline 10 & 96 & 99 & 97 & 100 & 92 & 11 \\
\hline
\end{tabular}

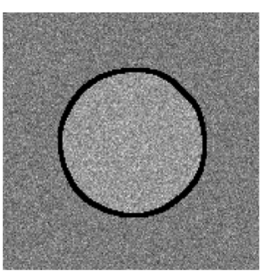

(a)

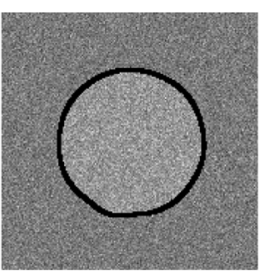

(b)

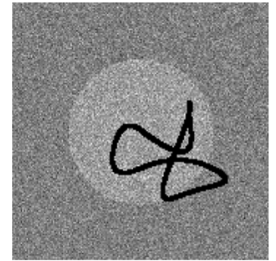

(c)
Fig. 9. Segmentation results for noisy synthetic data with $\mathrm{SNR}=-5 \mathrm{~dB}$. (a) Barely accepted with $J=0.853 \%$. (b) Barely rejected with $J=1.001 \%$. (a) Rejected with $J=81.065 \%$.

In the second experiment, we investigated the sensitivity to noise of our snake depending on the number of snake coefficients $M$. We generated 100 noisy realizations of a circle with a radius of 50 pixel units for different SNRs. We computed the power of the noise over a region of interest of size $200 \times 200$. We illustrate a realization of the resulting images in the header of Table II.

We show the percentage of success in Table II. We considered that our snake succeeded in segmenting the circle when the optimization process led to a segmentation with $J<1 \%$. This criterion is very conservative, as shown in Fig. 9. We observe from the results that our snake is robust against noise since it is capable of giving a proper segmentation even for low SNRs. Furthermore, the increased sensitivity to noise as we increase the number of vector coefficients $M$ corresponds to the appearance of additional noise-related local minima in the energy of the snake. Therefore, $M$ should be chosen as small as possible in order to avoid overfitting of the noise but should be big enough to be able to approximate the shape of interest.

\section{Medical Data}

Now, we move away from synthetic data. We compare our snake against other snake variants in terms of accuracy and speed. We quantify their accuracy at outlining the endocardial wall of the left ventricle within slices of 3-D cardiac MR image sequences.

The data we used are short-axis cardiac MR image sequences from 33 subjects acquired in the Department of Imaging of the Hospital for Sick Children in Toronto, Canada [37]. For each subject, data consist of a time series of 20 volumes. For each volume, the number of slices varies from 8 to 15 . Each slice is a $256 \times 256$ image with a pixel spacing between 0.93 and $1.64 \mathrm{~mm}$. The ground truth was obtained by manual annotation. In each segmented image, 1000 points (called landmark points) define a closed polygon outlining the endocardial wall.

1) Accuracy: For each subject, we selected one slice guided by its anatomical structures along the long axis and its timing in the cardiac cycle. Since the region of interest is nearly elliptical, we used the minimalistic elliptical active contour named Ovuscule to provide a first estimate of the location and the orientation of the left ventricle [28]. Then, we refined the segmentation of the endocardial wall using the general parametric active contour model (1) for different values of $M$ and several basis functions. More specifically, we used linear and quadratic B-splines, our function (8) that we refer to as a third-order exponential spline, and an extended version of (8) that we refer to as a fourth-order exponential spline. The linear B-spline basis function has a smaller support than our function (8). However, it can only adopt the form of polygons. The quadratic B-spline basis function has the same support and regularity than (8). However, 


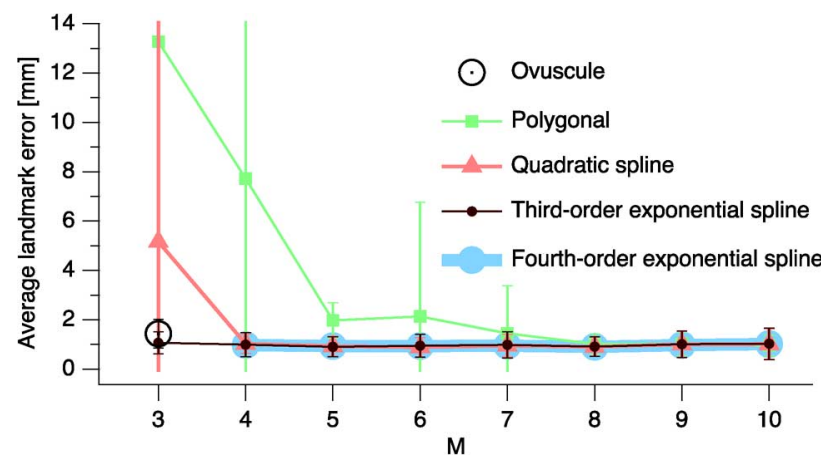

Fig. 10. Mean and variance of the landmark error across all 33 patients.

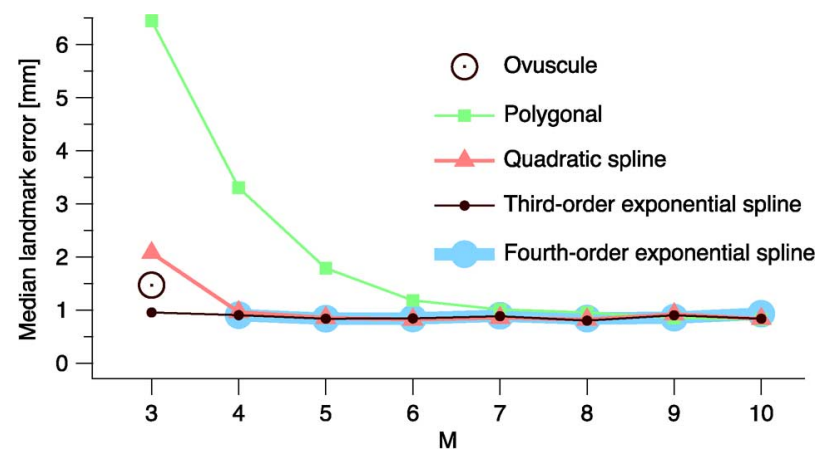

Fig. 11. Median of the landmark error across all 33 patients.

it is unable to reproduce ellipses. Finally, the fourth-order exponential spline is an extended version of (8), with one more degree of regularity but with a support of one unit larger. The initialization provided by the Ovuscule could be carried over to (8) and to the fourth-order exponential spline. In the case of the other types of snake, the perfect ellipse of the Ovuscule cannot be reproduced but must be approximated. This approximation was achieved by sampling the outline of the Ovuscule.

In a preprocessing step, the images were magnified four times horizontally and vertically. First, we evolved the Ovuscule on the magnified image. Second, we evolved more refined that were guided exclusively by the edge energy on a smoothed version of the magnified image. The smoothing was Gaussian, with a kernel of variance $\sigma^{2}=10^{2}$. We then measured the landmark error. We computed this error as the mean distance of the snake to the landmark points given by the ground truth, as was done in [37].

In Figs. $10-12$, we show the mean, median, and maximum values of the landmark error, respectively. From these graphs, we validate that the Ovuscule provides a good and robust starting point to be refined by the snakes investigated in this paper. The polygonal snake does not reach the accuracy of the Ovuscule until $M=7$ and exhibits a high variance across subject. The quadratic-spline snake and the third-order exponential-spline snake converge to similar accuracy starting with $M=4$. This was expected since we showed in Section III-B that our function does converge to a quadratic B-spline when $M$ increases. However, for low values of $M$, the difference is noticeable, and the quadratic-spline snakes produce shapes that are not compatible with the region of interest. Finally,

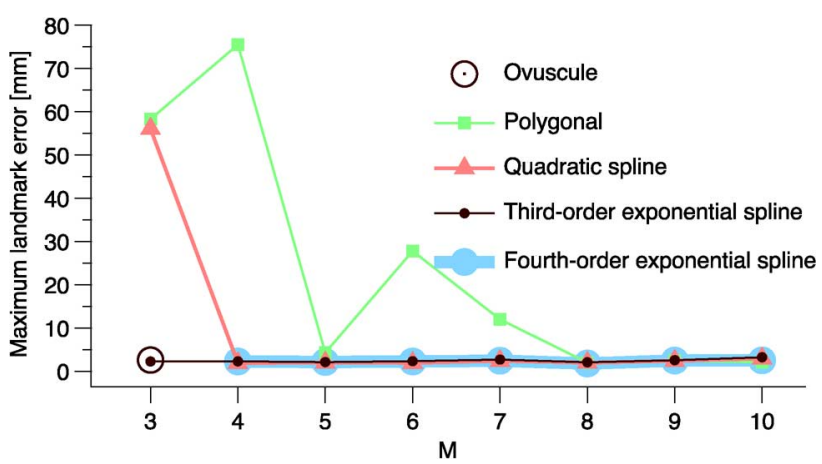

Fig. 12. Maximum landmark error among all 33 patients.

the fourth-order exponential-spline snakes produce equivalent results in terms of accuracy and stability than the third-order exponential-spline snake, at a price of a larger support, and therefore, of a slower convergence.

In Fig. 13(b), we illustrate the initialization provided to the Ovuscule and, in Fig. 13(c), the outcome of optimizing the Ovuscule, which will provide the initialization for further processing. We also show the result of several more elaborated snake variants and how they compare with the ground truth. The fourth-order exponential-spline snake results in an outline that is visually indistinguishable from that of the third-order one but comes at an increased computational cost.

2) Speed: In terms of speed, we compared our proposed snake to some classic traditional snakes such as a Kass-like snake [38] and a traditional geodesic active contour (GAC) model [13].

In this analysis, we used the anatomical structures of the 33 patients found in Section V-C1. However, we modified our initialization procedure to accommodate for the GAC model since it fails unless the initial contour lies totally inside or outside of the boundary of interest. Therefore, we scaled down the initialization that was provided by the outcome of optimizing the Ovuscule in Section V-C1. By doing so, we guarantee that all initial contours lay inside the endocardial wall to segment. Unfortunately, neither the Kass-like snake nor the GAC model are able to reproduce the initial ellipse perfectly, and their initialization must be approximated. This approximation was achieved by sampling the outline of the Ovuscule. Finally, we refined the segmentation of the endocardial wall by using our snake model for different values of $M$, the Kass-like snake, or the GAC.

This experiment was performed on a MacPro 3.1 with two Quad-Core Intel Xeon processors and 8 GB of RAM running Mac OS X 10.6.8. The implementation of the Kass-like active contour was taken from [38] and one of the GAC model from the free open-source image-processing package Fiji ${ }^{1}$ implementing the algorithm described in [13].

In Fig. 14, we show the mean temporal evolution of the improvement of the Jaccard distance during the snake evolution process for the 33 patients. We can clearly see that the proposed snake reaches its optimum earlier than the classical Kass-like snake and the GAC model. The Kass-like snake has a very costly first step. Moreover, it cannot escape a local minimum. The

\footnotetext{
${ }^{1}$ http://fiji.sc/
} 


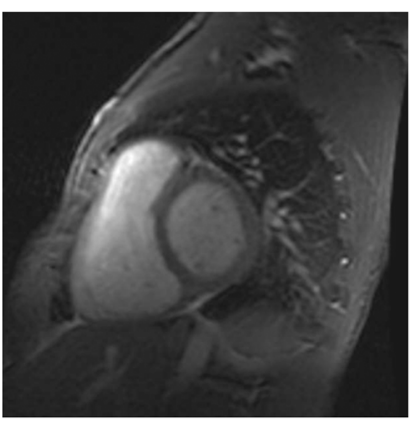

(a)

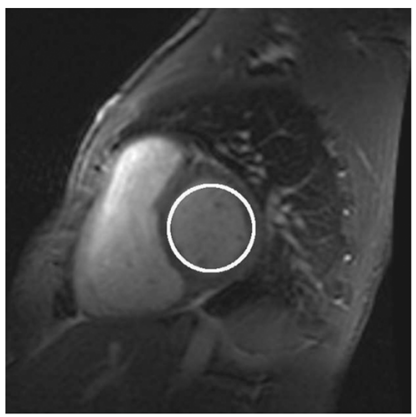

(b)

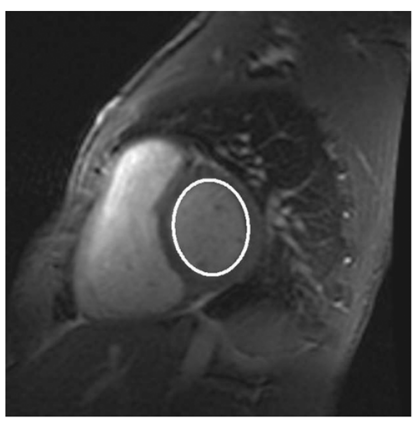

(c)

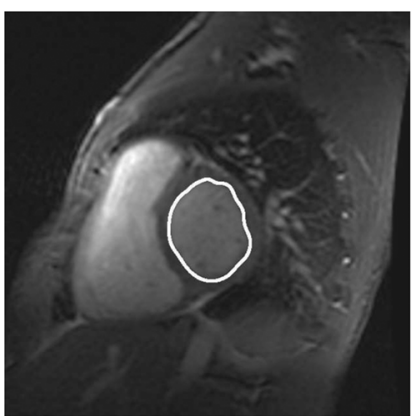

(d)

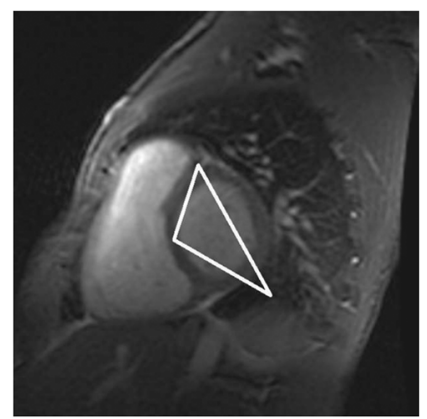

(e)

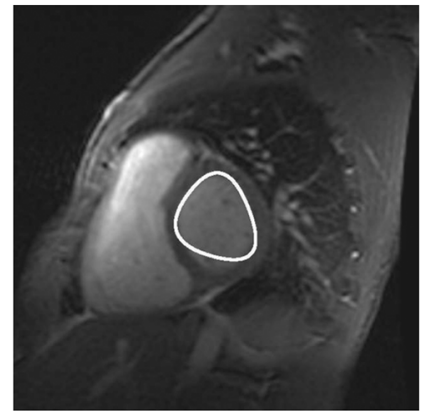

(f)

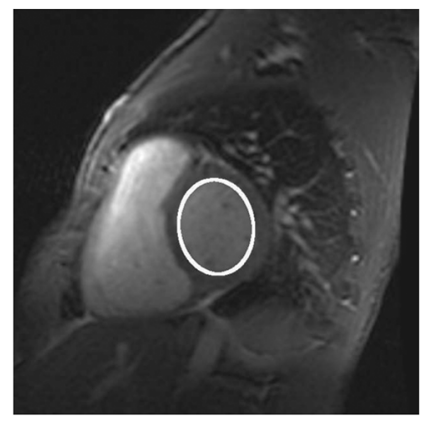

(g)

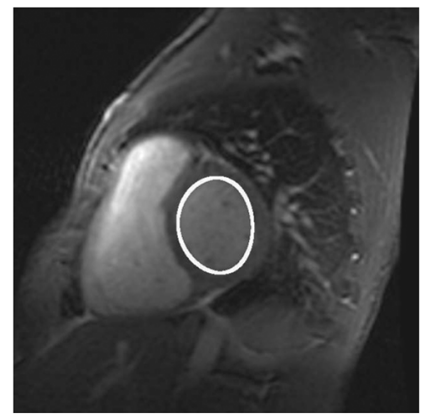

(h)
Fig. 13. Outline of the endocardial wall in the first frame and fourth slice of the second patient. (a) Raw data. (b) Initialization. (c) Ovuscule. (d) Ground truth. (e) Polygonal snake with $M=3$. (f) Quadratic-spline snake with $M=3$. (g) Third-order exponential-spline snake with $M=3$. (h) Fourth-order exponential-spline snake with $M=4$.

GAC is executed with an advection value of 2.2 and a propagation value of 1 . These parameters make the GAC succeed in overcoming the local minimum, but the convergence rate is still slower than that of the parametric case. It is important to notice that, for our proposed model, an increase in the number $M$ of control points slows the convergence. As pointed out in Section IV-D, this is due to the fact that the larger values of $M$ increase the computational load per iteration of the snake.

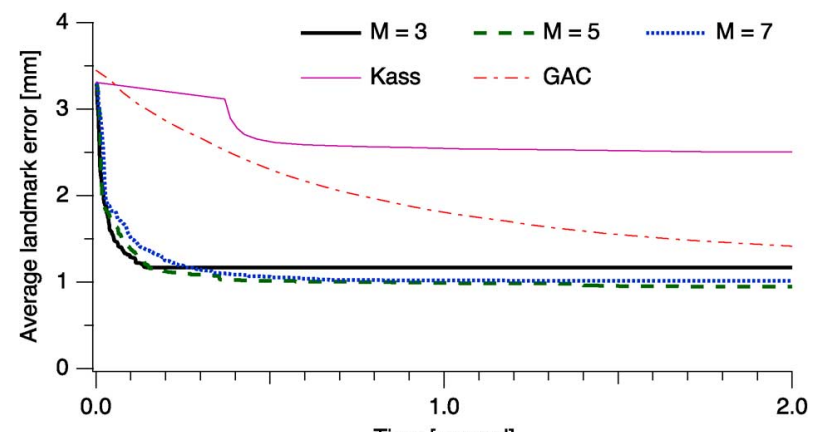

Fig. 14. Temporal evolution of the Jaccard distance. During the $2 \mathrm{~s}$ of snake evolution, the proposed method with $M=3$ performed 1479 iterations; with $M=5$, it performed 1406 iterations; and with $M=3$ it performed 889 iterations. The Kass snake performed 17 iterations. The first of these iterations took $370 \mathrm{~ms}$. The GAC performed 34 iterations.

\section{Real Data}

Here, we illustrate the behavior of our snake and provide further insights into its capabilities. In the context of this section, the ground truth is missing; therefore, we must relinquish quantitative assessments in favor of qualitative ones.

1) HeLa Nuclei: We want to evaluate the success of our snake model at outlining ellipse-like targets in the context of automated time-lapse microscopy. We use $(434 \times 434)$ images of HeLa nuclei that express fluorescent core histone $2 \mathrm{~B}$ on an RNAi live cell array. We show in Fig. 15 the result of the optimization process with (8) and $M=5$. This number of points is high enough to capture small departures from an elliptic shape.

We initialized every snake as a circle with a radius of 25 pixel units, as shown in Fig. 15. These initial circles were centered on the locations given by a maxima detector applied over a version of the image that was smoothed with a Gaussian kernel of variance $\sigma^{2}=12^{2}$ pixel. A total number of 23 maxima were detected. We then proceed with an inverted version of the original unsmoothed image to optimize the snakes. The optimization process converged in 22 cases. We show in Fig. 15 the result of the outlining process. We observe that our snakes were successful in most of the cases.

2) Droplets: As a second example, we show the outline of sprayed and deformed water droplets hitting a surface. The flight and the impact of the droplet was captured by a high-speed camera (Photron Fastcam) at a rate of 10000 images/s. The shape of the droplet is changing during flight, at impact, and while bouncing. After cropping, the size of the image was 663 $\times 663$ pixels.

We analyzed two frames. One was an image taken before the collision took place; the other was taken after the impact. In both cases, we initialized the snake as a circle with a position and size that we chose manually. These initializations are shown in Fig. 16. In the image prior to the impact, which we show in the left part of Fig. 16, a snake with $M=5$ was used. We selected a small value for $M$ because the droplet is nearly circular. In the image after the impact, which we show in the right part of Fig. 16, five control points did not provide enough freedom to cope with the discontinuity created by the attachment to the surface. However, the outline was successfully retrieved when slightly increasing the number of nodes to $M=8$. 


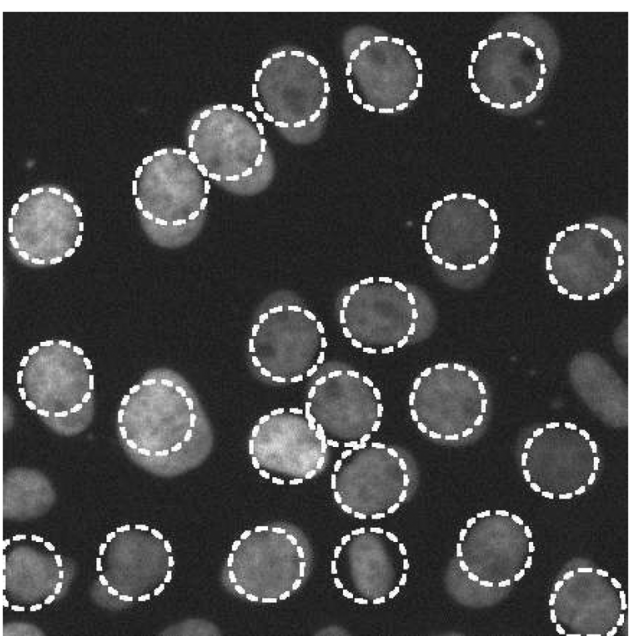

(a)

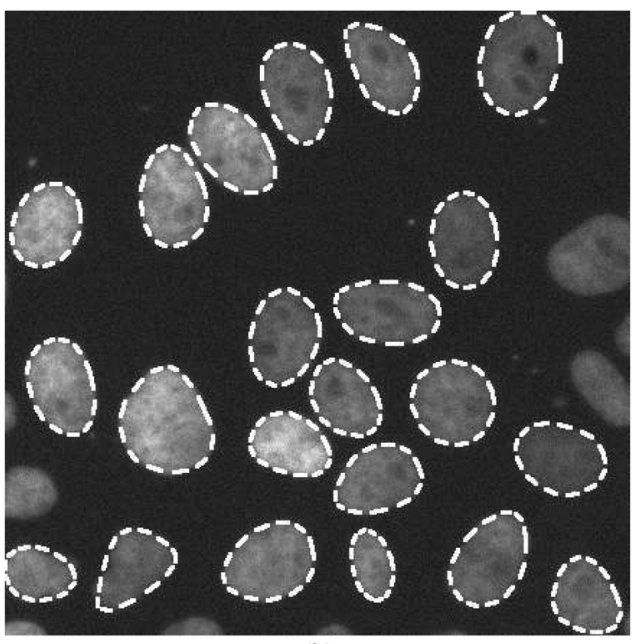

(b)

Fig. 15. Outline of HeLa nuclei in a fluorescence microscopy image. The parametric snakes were built with $M=5$. (a) Initial contour of the snake. (b) Result provided by our snake.

The method described in this paper has been programmed as a plugin for ImageJ, which is a free open-source multiplatform Java image-processing software. ${ }^{2}$ Our plugin $^{3}$ is independent of any imaging hardware, and due to ImageJ, any common file format may be used.

\section{CONCLUSION}

Our contribution in this paper is a new family of basis functions that we have used to describe parametric contours in terms of a set of control points. We were able to single out the basis of shortest support that allows one to reproduce circles and ellipses. Those can be characterized exactly by as few as three control points, but by considering additional ones, our parametric contours can reproduce with arbitrary precision any planar closed curve. In particular, we have shown that the mean error of approximation decays in the inverse proportion of the cube of the number of control points. We have used our ellipse-reproducing parametric curves to build snakes driven

\footnotetext{
2 http://rsb.info.nih.gov/ij/

${ }^{3}$ http://bigwww.epfl.ch/algorithms/esnake/
}

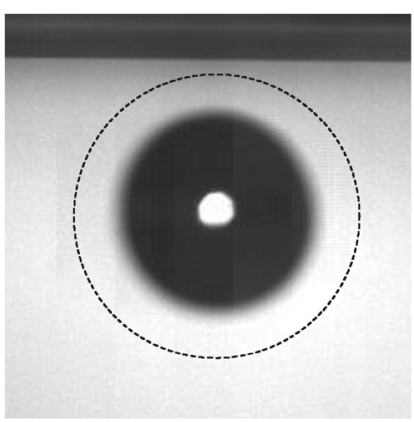

(a)

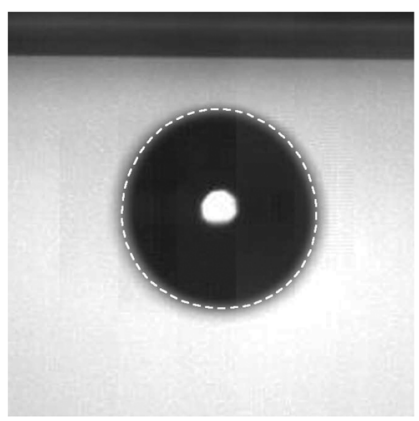

(c)

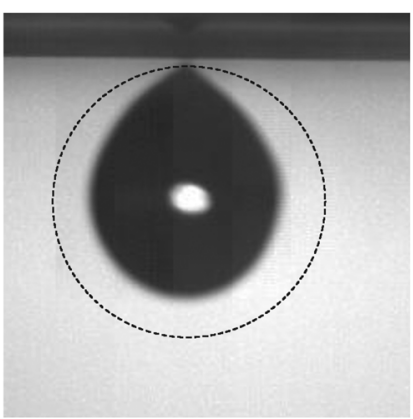

(b)

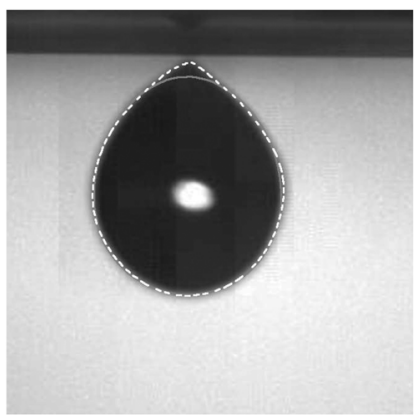

(d)
Fig. 16. Sprayed droplets. (a) Prior to the impact: The initial contour of the snake is is represented with a black dashed line. (b) After the impact: The initial contour of the snake it is represented with a black dashed line. (c) Prior to the impact: The outline of our snake with $M=5$ is represented with a white dashed line. (d) After the impact: The outline of the successful snake it is represented with a white dashed line $(M=8)$, while the configuration with $M=5$ is represented with a gray solid line. The droplet edges are partially out of focus, making them blurry and noisy.

by a combination of contour-based and region-based energies. In the latter case, the energy depends on the contrast between two regions: one being delineated by the curve itself and the other by an ellipse of double area. To determine this ellipse, we showed how to compute the best elliptical approximation, in a least-squares sense, of a contour described by an arbitrary number of control points. We were able to accelerate the implementation of our snakes by taking advantage of Green's theorem, which was facilitated by the availability of the explicit expressions of our basis. We have applied our snakes to a variety of problems that involve synthetic simulations and real data. We achieved excellent objective and subjective performance.

\section{REFERENCES}

[1] T. McInerney and D. Terzopoulos, "Deformable models in medical image analysis: A survey," Med. Image Anal., vol. 1, no. 2, pp. 91-108, Jun. 1996.

[2] A. Jain, Y. Zhong, and M.-P. Dubuisson-Jolly, "Deformable template models: A review," Signal Process., vol. 71, no. 2, pp. 109-129, Dec. 1998.

[3] M. Jacob, T. Blu, and M. Unser, "Efficient energies and algorithms for parametric snakes," IEEE Trans. Image Process., vol. 13, no. 9, pp. 1231-1244, Sep. 2004.

[4] M. Kass, A. Witkin, and D. Terzopoulos, "Snakes: Active contour models," Int. J. Comput. Vis., vol. 1, no. 4, pp. 321-331, Jan. 1988.

[5] C. Xu and J. Prince, "Snakes, shapes, and gradient vector flow," IEEE Trans. Image Process., vol. 7, no. 3, pp. 359-369, Mar. 1998.

[6] J. Gao, A. Kosaka, and A. Kak, "A deformable model for human organ extraction," in Proc. IEEE ICIP, 1998, pp. 323-327.

[7] L. Staib and J. Duncan, "Boundary finding with parametrically deformable models," IEEE Trans. Pattern Anal. Mach. Intell., vol. 14, no. 11, pp. 1061-1075, Nov. 1992. 
[8] A. Chakraborty, L. Staib, and J. Duncan, "Deformable boundary finding in medical images by integrating gradient and region information," IEEE Trans. Med. Imag., vol. 15, no. 6, pp. 859-870, Dec. 1996.

[9] M. Figueiredo, J. Leitão, and A. Jain, "Unsupervised contour representation and estimation using B-splines and a minimum description length criterion," IEEE Trans. Image Process., vol. 9, no. 6, pp. 1075-1087, Jun. 2000.

[10] P. Brigger, J. Hoeg, and M. Unser, "B-spline snakes: A flexible tool for parametric contour detection," IEEE Trans. Image Process., vol. 9, no. 9, pp. 1484-1496, Sep. 2000.

[11] M. Gebhard, J. Mattes, and R. Eils, "An active contour model for segmentation based on cubic B-splines and gradient vector flow," in Proc. Conf. MICCAI, 2001, vol. 2208, pp. 1373-1375.

[12] R. Malladi, J. Sethian, and B. Vemuri, "Shape modeling with front propagation: A level set approach," IEEE Trans. Pattern Anal. Mach. Intell., vol. 17, no. 2, pp. 158-175, Feb. 1995.

[13] V. Caselles, R. Kimmel, and G. Sapiro, "Geodesic active contours," Int. J. Comput. Vis., vol. 22, no. 1, pp. 61-79, Feb./Mar. 1997.

[14] K. Zhang, L. Zhang, H. Song, and W. Zhou, "Active contours with selective local or global segmentation: A new formulation and level set method," Image Vis. Comput., vol. 28, no. 4, pp. 668-676, Apr. 2010.

[15] K. Zhang, H. Song, and L. Zhang, "Active contours driven by local image fitting energy,” Pattern Recognit., vol. 43, no. 4, pp. 1199-1206, Apr. 2010.

[16] T. McInerney and D. Terzopoulos, "T-snakes: Topology adaptive snakes," Med. Image Anal., vol. 4, no. 2, pp. 73-91, Jun. 2000.

[17] F. Precioso and M. Barlaud, "B-spline active contour with handling of topology changes for fast video segmentation," EURASIP J. Appl. Signal Process., vol. 2002, no. 6, pp. 555-560, Jun. 2002.

[18] G. Sapiro, Geometric Partial Differential Equations and Image Analysis, 1st ed. Cambridge, U.K.: Cambridge Univ. Press, 2006.

[19] J. Blokland, A. Vossepoel, A. Bakker, and E. Pauwels, "Delineating elliptical objects with an application to cardiac scintigrams," IEEE Trans. Med. Imag., vol. MI-6, no. 1, pp. 57-66, Mar. 1987.

[20] P. Lipson, A. Yuille, D. O'Keeffe, J. Cavanaugh, J. Taaffe, and D. Rosenthal, "Deformable templates for feature extraction from medical images," in Proc. 1st ECCV, 1990, pp. 413-417.

[21] I. Adame, R. Van Der Geest, D. Bluemke, J. Lima, J. Reiber, and B. Lelieveldt, "Automatic vessel wall contour detection and quantification of wall thickness in in-vivo MR images of the human aorta," J. Magn. Reson. Imag., vol. 24, no. 3, pp. 595-602, Sep. 2006.

[22] J. Guerrero, S. Salcudean, J. McEwen, B. Masri, and S. Nicolaou, "Real-time vessel segmentation and tracking for ultrasound imaging applications," IEEE Trans. Med. Imag., vol. 26, no. 8, pp. 1079-1090, Aug. 2007.

[23] S. Tsuji and F. Matsumoto, "Detection of ellipses by a modified Hough transformation," IEEE Trans. Comput., vol. C-27, no. 8, pp. 777-781, Aug. 1978.

[24] N. Bennett, R. Burridge, and N. Saito, "A method to detect and characterize ellipses using the Hough transform," IEEE Trans. Pattern Anal. Mach. Intell., vol. 21, no. 7, pp. 652-657, Jul. 1999.

[25] I. Adame, R. van der Geest, B. Wasserman, M. Mohamed, J. Reiber, and B. Lelieveldt, "Automatic segmentation and plaque characterization in atherosclerotic carotid artery MR images," Magn. Reson. Mater. Phys., Biol. Med., vol. 16, no. 5, pp. 227-234, Apr. 2004.

[26] B. Alberts, A. Johnson, J. Lewis, M. Raff, K. Roberts, and P. Walter, Molecular Biology of The Cell, 5th ed. New York: Garland Science, 2007, $1601 \mathrm{p}$

[27] A. Stalder, G. Kulik, D. Sage, L. Barbieri, and P. Hoffmann, "A snakebased approach to accurate determination of both contact points and contact angles," Colloids Surf. A: Physicochem. Eng. Aspects, vol. 286, no. 1-3, pp. 92-103, Sep. 2006.

[28] P. Thévenaz, R. Delgado-Gonzalo, and M. Unser, "The ovuscule," IEEE Trans. Pattern Anal. Mach. Intell., vol. 33, no. 2, pp. 382-393, Feb. 2011

[29] M. Unser, "Sampling-50 years after Shannon," Proc. IEEE, vol. 88, no. 4, pp. 569-587, Apr. 2000.

[30] M. Jacob, T. Blu, and M. Unser, "Sampling of periodic signals: A quantitative error analysis," IEEE Trans. Signal Process., vol. 50, no. 5, pp. 1153-1159, May 2002.

[31] A. Aldroubi and M. Unser, "Sampling procedures in function spaces and asymptotic equivalence with Shannon's sampling theory," Numer. Funct. Anal. Optim., vol. 15, no. 1/2, pp. 1-21, 1994.

[32] R. Delgado-Gonzalo, P. Thévenaz, and M. Unser, "Exponential splines and minimal-support bases for curve representation," Comput. Aided Geometric Des., accepted for publication.
[33] M. Unser and T. Blu, "Cardinal exponential splines: Part I-Theory and filtering algorithms," IEEE Trans. Signal Process., vol. 53, no. 4, pp. 1425-1438, Apr. 2005.

[34] M. Unser, "Splines: A perfect fit for signal and image processing," IEEE Signal Process. Mag., vol. 16, no. 6, pp. 22-38, Nov. 1999.

[35] M. Jacob, T. Blu, and M. Unser, "A unifying approach and interface for spline-based snakes," Progress in Biomedical Optics and Imaging, vol. 2, no. 27. San Diego, CA, vol. 4322, Part I, 2001, pp. 340-347.

[36] W. Press, S. Teukolsky, W. Vetterling, and B. Flannery, Numerical Recipes: The Art of Scientific Computing, 3rd ed. Cambridge, U.K.: Cambridge Univ. Press, 1986.

[37] A. Andreopoulos and J. Tsotsos, "Efficient and generalizable statistical models of shape and appearance for analysis of cardiac MRI," Med. Image Anal., vol. 12, no. 3, pp. 335-357, Jun. 2008.

[38] P. Andrey and T. Boudier, "Adaptive active contours," in Proc. 1st ImageJ User Developer Conf., Luxembourg, 2006.

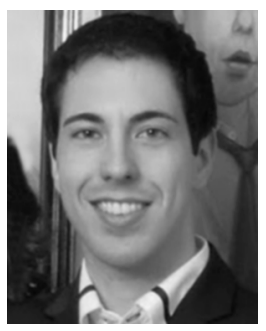

Ricard Delgado-Gonzalo was born in Barcelona, Spain, in 1983. He received the Diploma in telecommunications engineering and the Diploma in mathematics from the Universitat Politècnica de Catalunya, Barcelona, in 2006 and 2007, respectively. $\mathrm{He}$ is currently working toward the Ph.D. degree with the Biomedical Imaging Group, École polytechnique fédérale de Lausanne (EPFL), Lausanne, Switzerland.

In 2008, he joined the Biomedical Imaging Group, EPFL, as a Ph.D. student and as an Assistant. He is currently working on applied problems related to image reconstruction, segmentation, and tracking, as well as on the mathematical foundations of signal processing and spline theory. His research interests include technology management and transfer.

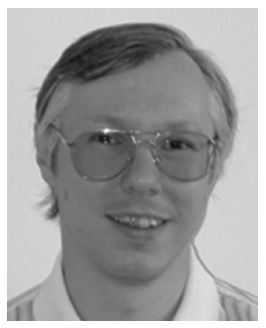

Philippe Thévenaz was born in Lausanne, Switzerland, in 1962. He received the Diploma in microengineering from the École polytechnique fédérale de Lausanne (EPFL), Lausanne, Switzerland, in 1986 and the Ph.D. degree with a thesis on the use of the linear prediction residue for text-independent speaker recognition.

Then, he joined the Institute of Microtechnology, University of Neuchâtel, Neuchâtel, Switzerland, where he worked in the domain of image processing (optical flow) and speech processing (speech coding and speaker recognition). Then, he worked as a Visiting Fellow with the Biomedical Engineering and Instrumentation Program, National Institutes of Health, Bethesda, MD. Since 1998, he has been with the EPFL as a Senior Researcher. His research interests include splines and multiresolution signal representations, geometric image transformations, and biomedical image registration.

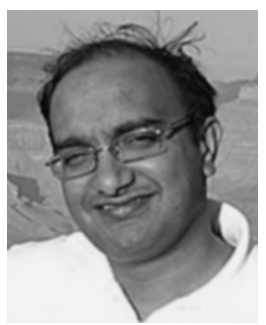

Chandra Sekhar Seelamantula (M'99) was born in Gollepalem, Andhra Pradesh, India, in 1976. He received the B.E. degree (with honors), with specialization in electronics and communication engineering, from the University College of Engineering, Osmania University, Hyderabad, India, in 1999 and the Ph.D. degree from the Indian Institute of Science, Bangalore, India, in 2005, with a thesis entitled Time-Varying Signal Models: Envelope and Frequency Estimation with Applications to Speech and Music Signal Compression.

During his doctoral years, he specialized in the development of auditorymotivated signal processing models for speech and audio applications. From April 2005 to March 2006, he worked as a Technology Consultant for M/s. ESQUBE Communication Solutions Pvt. Ltd., Bangalore, and developed proprietary audio coding solutions. In April 2006, he joined the Biomedical Imaging Group, École polytechnique fédérale de Lausanne, Lausanne, Switzerland, as a Postdoctoral Fellow and specialized in the field of image processing, optical coherence tomography, holography, splines, and sampling theories. Since July 2009, he has been an Assistant Professor with the Department of Electrical Engineering, Indian Institute of Science. 


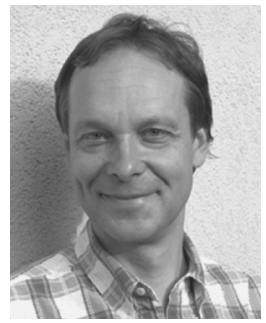

Michael Unser (M'89-SM'94-F'99) received the M.S. (summa cum laude) and Ph.D. degrees in electrical engineering from the École Polytechnique Fédérale de Lausanne, Lausanne, Switzerland, in 1981 and 1984, respectively.

From 1985 to 1997 , he worked as a Scientist with the National Institutes of Health, Bethesda, MD. He is currently a Full Professor and a Director of the Biomedical Imaging Group, EPFL. He is the author of 200 journal papers and is one of ISI's Highly Cited authors in Engineering ( http://isihighlycited.com).

His main research interest include biomedical image processing. His other main interests include sampling theories, multiresolution algorithms, wavelets, and the use of splines for image processing.
Dr. Unser served as an Associate Editor-in-Chief for IEEE TRANSACTIONS ON MEDICAL IMAGING in 2003-2005 and as an Associate Editor for the same journal in 1999-2002 and in 2006-2007. He also served as an Associate Editor for the IEEE TRANSACTIONS ON IMAGE PROCESSING in 1992-1995 and for the IEEE SIGNAL PROCESSING LETTERS in 1994-1998. He is currently a member of the editorial boards of Foundations and Trends in Signal Processing, and Sampling Theory in Signal and Image Processing. He coorganized the first IEEE International Symposium on Biomedical Imaging and was the Founding Chair of the technical committee of the IEEE-SP Society on Bio-Imaging and Signal Processing. He was the recipient of the 1995 and 2003 Best Paper Awards, the 2000 Magazine Award, and two IEEE Technical Achievement Awards (2008 SPS and 2010 EMBS). He is an EURASIP Fellow and a member of the Swiss Academy of Engineering Sciences. 